\title{
A CORRELAÇÃO ENTRE NÍVEL DE PROFICIÊNCIA E DESEMPENHO NA IDENTIFICAÇÃO DA PLOSIVA ALVEOLAR ASPIRADA DO INGLÊS (L2)
}

\author{
The correlation between proficiency level and performance in the identification of \\ the English-L2 alveolar aspirated plosive
}

Felipe Flores KUPSKE, UNOCHAPECÓ ${ }^{1}$

\begin{abstract}
RESUMO: Neste trabalho, tentamos verificar a existência de correlação entre nível de proficiência em L2 e média de acertos na identificação da plosiva alveolar aspirada do inglês. Contamos com 14 participantes palatalizantes aprendizes de inglês (L2) do Oeste de Santa Catarina, e nosso teste de identificação foi elaborado com estímulos de um locutor nativo do inglês. A testagem apontou uma associação significativa entre proficiência e desempenho. Contudo, nossa testagem revela que a diferença é significativa apenas na média de erro em função da proficiência quando os segmentos antecediam [i:]. Nesta perspectiva, podemos pensar que os informantes de nível básico de proficiência em inglês identificam os segmentos testados como tokens de um mesmo som nativo, isto é, categorizariam africada e plosiva aspirada antes de vogal alta não arredondada em uma mesma categoria.
\end{abstract}

PALAVRAS-CHAVE: Aspiração; Identificação; Nível de proficiência; Língua Inglesa

ABSTRACT: In this study we tried to assess the existence of correlation between level of proficiency and performance in the identification of the English-L2 alveolar aspirated plosive. The studied counted with 14 learners of English (L2) that present palatalization in Portuguese, and our identification test was prepared with stimuli from a native speaker of English. The testing indicated a significant correlation between proficiency and performance. However, our testing shows that the difference is significant when the segments precede [i:]. In this perspective, we think that the basic informants identify the segments tested as tokens of the same native sound, that is, affricate and plosive aspirated before high rounded vowel would belong in the same category.

KEY-WORDS: Aspiration, Identification; Proficiency level, English

\section{INTRODUÇÃO}

Estudos recentes têm apontado que a fala - por muitos anos às margens dos estudos científicos da linguagem - não apenas é central para a linguística, como seria, acima de tudo, o próprio locus da aquisição da linguagem. Nesse sentido, podemos observar, também, que os estudos de aquisição da oralidade, que outrora apenas tentavam elucidar questões de produção da fala, têm se debruçado, cada vez mais, em estudos balizados por

\footnotetext{
${ }^{1}$ Professor titular da Universidade Comunitária da Região de Chapecó (UNOCHAPECÓ) e estudante de doutorado em Linguística Aplicada (Aquisição da Linguagem) - UFRGS.
} 
questões de percepção dos planos de expressões da comunicação humana. Modelos como, por exemplo, o Perceptual Assimilation Model - L2 (doravante PAM-L2 - BEST; TYLER, 2007) versam sobre a militância das categorias fonético-fonológicas de L1 na percepção e, consequentemente, na produção dos sons de L $2^{2}$. Tais apontamentos fazem com que questionamentos e, por conseguinte, investigações que se debrucem na percepção humana venham à tona. A presente empreitada enquadra-se neste grupo de corrente de investigação.

Para os que advogam modelos linguísticos que contemplam o gesto ${ }^{3}$ como unidade de análise, parece haver um consenso de que falantes de diferentes sistemas de L1, ao retirarem probabilisticamente padrões de sua comunidade de fala, apresentariam diferentes pautas gestuais para a percepção e produção de sons (ALBANO, 2001). Sabese, ainda, que a fricção nas plosivas coronais antes de vogal alta [i] possui um alto índice de aplicação para os falantes do oeste de Santa Catarina (GUZZO; BATISTTI, 2009). Destarte, podemos pensar que as pautas gestuais da plosiva coronal /t/ destes falantes em contexto que precede a vogal alta [i] sejam diferentes de pautas para os demais contextos da distribuição complementar da plosiva nos quais a africada não se aplica. Partindo do pressuposto que as africadas diferenciam-se dos demais sons consonantais pela presença de um ruído acústico (chiamento) e que estão presentes, em Português Brasileiro (PB), apenas antes de [i] (ALBANO, 2001), resolvemos analisar como falantes palatalizantes performariam na identificação de sons de uma L2 que apresentassem ruídos acústicos próximos aos das africadas do PB. Nesta perspectiva, neste trabalho, ainda embrionário, procuramos investigar a percepção da aspiração nas plosivas surdas do inglês por falantes palalizantes da região do Oeste de Santa Catarina (SC), compreendendo a região de Chapecó e seu entorno. É válido destacar que um dado empírico que fomenta a nossa curiosidade para a elaboração deste trabalho é o fato de aprendizes brasileiros de inglêsL2 produzirem [t $\mathrm{fu}:$, com a presença da africada surda antes de vogal alta arredondada, para o item two, [dois] do inglês, que demandaria a produção de uma plosiva coronal aspirada, como em [ $\left.\mathrm{t}^{\mathrm{h}} \mathrm{u}:\right]$. Esse exemplo é usado para atestar que poderia haver, em algum

\footnotetext{
${ }^{2}$ Este trabalho não prevê diferenciação entre os termos Língua Estrangeira e Segunda Língua. Esses são usados intercambiavelmente ao longo deste trabalho.

${ }^{3}$ Segundo Silva (2003), o termo gesto articulatório não é novo na literatura da fonética. No entanto, fora da Fonologia Articulatória (FAR), ele é concebido simplesmente como um movimento de um articulador. $\mathrm{Na}$ FAR, assim como neste trabalho, o termo tem outro sentido: é a representação de todas as manobras articulatórias necessárias para se realizar um determinado som da fala.
} 
nível, um processo de transferência resultante do contato entre categorias fonéticofonológicas de aspiração e de africação.

Destarte, mais especificamente, procuramos analisar, neste trabalho, se falantes palatalizantes obterão índices lineares na identificação da plosiva alveolar surda aspirada do inglês em contextos vocálicos variados, focando na existência ou não de uma correlação estatisticamente significativa entre o nível de proficiência em inglês-L2 e a média de acertos na tarefa de identificação da plosiva alveolar surda aspirada do inglês.

\section{A TRANSFERÊNCIA FONÉTICO-FONOLÓGICA}

No que concerne à fala, a transferência fonético-fonológica faz referência à utilização de padrões já estabelecidos, ou engramados, da L1 nas produções de L2. Partindo do pressuposto que aprendizes de L2 já possuem um sistema cognitivo completo e operante em L1, aspectos fonéticos e fonológicos dessa L2 se conectam com o sistema de L1, mantendo relações de similaridade e diferença (FLEGE, 1995). Desta forma, em uma perspectiva dinâmica da linguagem, a transferência de padrões é uma estratégia de aprendizagem inerente ao próprio processo, tendo em vista que o cérebro trabalha em paralelo, isto é, de maneira interconectada. A aquisição da L2 começa fortemente influenciada pela L1 - sistema cognitivo altamente organizado. Contudo, segundo MacWhinney (2002), essa forte associação entre L1 e L2 vai, aos poucos, desfazendo-se, mesmo que uma possível transferência residual permaneça.

Em um prisma dinâmico, o conceito de transferência está imbricado ao conceito de entrincheiramento (ZIMMER, 2004, 2008; ZIMMER; ALVES, 2006; SEIDENBERG; ZEVIN, 2006; MACWHINNEY, 2006). Para MacWhinney (2006), quando uma tarefa é praticada diversas vezes, pode-se esperar que ela seja automatizada ou entrincheirada e, quanto maior o uso efetivo dessa tarefa, mais profundo seria esse entrincheiramento. Assim, espera-se que a força ou robustez de uma L1 seja maior do que a da L2 em processo de aquisição, e essa, consequentemente, será transferida em seu processamento, o que, em fonologia, representa, por exemplo, o sotaque estrangeiro, uma vez que os sons da L2 podem ser processados por sons similares da L1.

Vários modelos foram propostos no que diz respeito à transferência e à aquisição da oralidade em L2. Aqui, contudo, destacamos dois desses modelos: o Speech Learning Model (FLEGE, 1995); e Perceptual Learning Model - L2 (BEST; TYLER, 2007). Para o primeiro modelo, Flege (1995) aponta que a percepção dos sons da L2 se dá através de 
um "filtro" da L1. Assim, quando um som da L2 é percebido como similar a um som da L1, aquele pode ser categorizado tal qual o som da L1. Para o autor (1995), esse processo ocorreria devido a dificuldades perceptivas das diferenças acústico-articulatórias entre a L1 e a língua-alvo. Portanto, quanto maior a proximidade fonético-fonológica dos sons envolvidos no processo de aquisição de L2, mais difícil serão as tarefas perceptivas e, como consequência, a produção desses segmentos. Desta forma, assim como apontam Zimmer, Silveira e Alves (2009), o Speech Learning Model prevê que as limitações de percepção são oriundas da experiência prévia dos aprendizes com sua própria L1.

O modelo de Best e Tyler (2007), o Perceptual Learning Model - L2, também contempla aspectos acústicos da aquisição e, segundo Alves et al. (2011), é mais explicitamente associada a uma concepção dinâmica e emergentista, aqui defendida. Para Zimmer, Silveira e Alves (2009), o modelo Perceptual Assimilation Model (BEST, 1995), antecessor do Perceptual Learning Model - L2, contempla a influência da L1 na restrição perceptual de fones não nativos, lançando luz não na percepção de aprendizes de línguas, mas dos nä̈ve monolinguals, isto é, aqueles falantes com pouco ou nenhuma experiência com a L2. Contudo, esse modelo, embora contemplasse o nível inicial do desenvolvimento fonético-fonológico da L2, pecava no quesito referente às suas etapas desenvolvimentais. Dessa forma, o modelo foi atualizado em 2007. Nesse modelo, o primitivo de análise é o gesto, isto é, os fones são percebidos por meio de gestos articulatórios, o que aproxima a teoria aos modelos dinâmicos da linguagem, como a Fonologia Articulatória (BROWMAN; GOLDSTEIN, 1992), por exemplo. Para o Perceptual Learning Model - L2, os sons da L1 e L2 em processo de aquisição coexistem em um espaço fonológico único, oferecendo, desta forma, contexto para a transferência, na qual o detalhe fonético é o elemento que constitui a representação linguística. Transferência é tida como a interação dos sistemas de L1 e L2 que coabitam este mesmo espaço fonológico (ALVES et al., 2011).

\section{A ASPIRAÇÃO DE PLOSIVAS SURDAS E O VOT}

Segundo Rothenber (2009), o termo Voice Onset Time (VOT) foi desenvolvido como um parâmetro para os padrões de um sintetizador da fala desenvolvido aproximadamente há 50 anos, para que este mecanismo pudesse diferenciar a produção das plosivas surdas e sonoras do inglês americano. Para que as diferenças percebidas no modo de articulação das plosivas fossem obtidas pelo sintetizador, um atraso era, às 
vezes, empregado entre a consoante e o som da vogal seguinte. Contudo, embora esse conceito tenha sido originado para tais fins e não como uma ferramenta de análise linguística, esse viés muda quando Lisker e Abramson (1964) começam a empregar essa terminologia para suas pesquisas em fonética.

Para Lisker e Abramson (1964), é senso comum em fonética afirmar que as categorias das plosivas em várias línguas podem ser divididas em grupos que diferem sobre o vozeamento: plosivas sonoras apresentam vibração glotal durante o intervalo da oclusão, enquanto a não presença dessa vibração é a marca das plosivas surdas. Ainda para os autores, espectrograficamente, os dois tipos de plosivas são, na maioria dos casos, facilmente distinguidas. Em termos acústicos, os segmentos sonoros apresentam energia de baixa frequência na região de seu primeiro formante. Por outro lado, as surdas apresentam um período de silêncio completo, dada a abertura da glotes, o que permite a passagem ininterrupta do ar fonatório (LISKER; ABRAMSON, 1964).

Contudo, embora essa diferença estabeleça uma base adequada para a separação de categorias de plosivas em inúmeras línguas do mundo, para outras línguas, como no caso do inglês e do alemão, por exemplo, essa diferença é eficiente apenas em parte. No inglês, por exemplo, os grupos /b, d, g/ e /p, t, k/ não podem ser distinguidos nitidamente nesta base. Em posição medial, /b, d, g/ são sonoras e /p, t, k/ é um grupo surdo, porém, em posição inicial, ambos os grupos são comumente produzidos com intervalos de closura silenciosa e poderiam, desta forma, ser considerados surdos (LISKER; ABRAMSON, 1964). Neste sentido, busca-se a aspiração para a identificação de cada um dos grupos, face ao fato de que /p, t, k/ liberam uma explosão de ar quando em posição inicias e /b, d, g/ não, como já mencionado. Destarte, o VOT seria capaz de separar as duas categorias das plosivas em diversas posições. Nesse sentido, os autores concluem que o VOT é relevante para diferenciar as plosivas em diversas línguas do mundo. Segundo Kessinger e Blumstein (1997), 88,9\% das línguas do homem utilizam o vozeamento como característica distintiva, sendo que o VOT é, então, uma pista acústica associada a tal distinção.

O VOT pode ser dividido em três categorias, segundo Lisker e Abramson (1964), sendo elas: a) VOT negativo, em que há vozeamento antes da barra de explosão de valor entre $-125 \mathrm{~ms}$ e $-75 \mathrm{~ms}$; b) VOT zero, que apresenta um período de surdez pequeno ou nulo, em que o início de vozeamento e a soltura ocorrem em um período bastante próximo, em torno de 0 a $10 \mathrm{~ms}$; e c) VOT positivo, que possui um intervalo longo entre 
a barra de explosão e o início do vozeamento, em torno de +75 ms. Esse último padrão é o que configura a aspiração.

Cho e Ladefoged (1999, p. 225) definem VOT a partir de uma perspectiva articulatória. Para os autores, VOT é intervalo de tempo entre o início do gesto articulatório responsável pela soltura e o início do gesto laringal, responsável pela vibração das cordas vocais. Os autores, então, propõem quatro categorias fonéticas para as plosivas surdas. A primeira refere-se às plosivass sem aspiração, com VOT em torno de $30 \mathrm{~ms}$ (0 - $40 \mathrm{~ms}$ ); a segunda em torno de $50 \mathrm{~ms}$ (40 - $60 \mathrm{~ms}$ ) para plosivas levemente aspiradas, a terceira para plosivas aspiradas em torno de $90 \mathrm{~ms}(60$ - $100 \mathrm{~ms})$ e, a última, para plosivas altamente aspiradas, em torno de $100 \mathrm{~ms}$ (100 - $160 \mathrm{~ms})$.

Embora não exista uma medida absoluta para valores de VOT para cada plosiva surda, Toribio et al. (2005) apontam que os valores típicos do inglês estão em torno dos $80 \mathrm{~ms}$ para /k/, $70 \mathrm{~ms}$ para /t/, e $55 \mathrm{~ms}$ para /p/. Para o português, segundo Istre (apud REIS et al. 2008), os valores de VOT variam em torno de $38 \mathrm{~ms}$ para /k/, $18 \mathrm{~ms}$ para /t/ e $12 \mathrm{~ms}$ para /p/. Novamente, devemos ressaltar que, muito embora os valores supracitados sejam os mais presentes na literatura da área, esses valores não são canônicos e que há muita variação nas médias para a produção das plosivas nas pesquisas atuais. Além disso, os valores de VOT variam dependendo do contexto fonológico em inglês e em PB, bem como de acordo com a taxa de elocução.

Em português do Brasil, segundo Reis e Nobre-Oliveira (2007), as plosivas surdas tendem a ser produzidas com um VOT positivo, mas curto em qualquer contexto, sendo que alguns autores dizem, inclusive, que as plosivas do PB se encaixam sob o padrão VOT Zero (REIS et al., 2007). Em inglês, por outro lado, o padrão de VOT para esse grupo de consoantes tende a ser positivo e longo em posição inicial de palavra e em sílabas tônicas.

Em relação a essa diferença de valores de VOT, para Cohen (2004), uma das principais características de um sotaque do inglês na pronúncia do PB é a aspiração de consoantes que não deveriam ser aspiradas. Da mesma forma, a dificuldade em aspirar as plosivas quando necessário pode contribuir para o sotaque em língua inglesa por um falante nativo do PB. Neste sentido, aspiração é parte importante no processo de percepção e produção das diferenças acústicas entre plosivas surdas sonoras entre falantes nativos do inglês. Contudo, grande parte dos aprendizes brasileiros do inglês - básicos ou mais avançados - não produzem a aspiração em posição inicial (ZIMMER, 2004; ZIMMER et al., 2009). 


\section{METODOLOGIA}

Esta seção contempla informações acerca dos participantes e do locutor do estudo, bem como os critérios de seleção dos estímulos adotados nos experimentos de percepção. Além disso, há a explicitação da tarefa de discriminação utilizada para a coleta de dados do presente trabalho.

\section{Participantes}

Aprendizes de língua inglesa, estudantes de graduação dos cursos de Letras de universidades da região oeste de Santa Catarina, foram convidados a participar da testagem. Os participantes interessados deveriam realizar o Oxford Placement Test 1 (ALLAN, 2004). Nesta primeira etapa, foram selecionados 14 estudantes, seis $(42,9 \%)$ classificados como básicos ou pertencentes (Grupo A) e oito $(57,1 \%$ ) classificados como intermediários avançados ou avançados (Grupo B). Dentre os informantes, três $(21,3 \%)$ são do sexo masculino, e o restante $(78,6 \%)$, do sexo feminino, e as idades variam entre 18 e 25 anos, com uma média total de 20,21 anos e um desvio padrão (DP) de 2,22 anos, conforme o Gráfico 1.

Gráfico 1 - Histograma da variável escalar idade

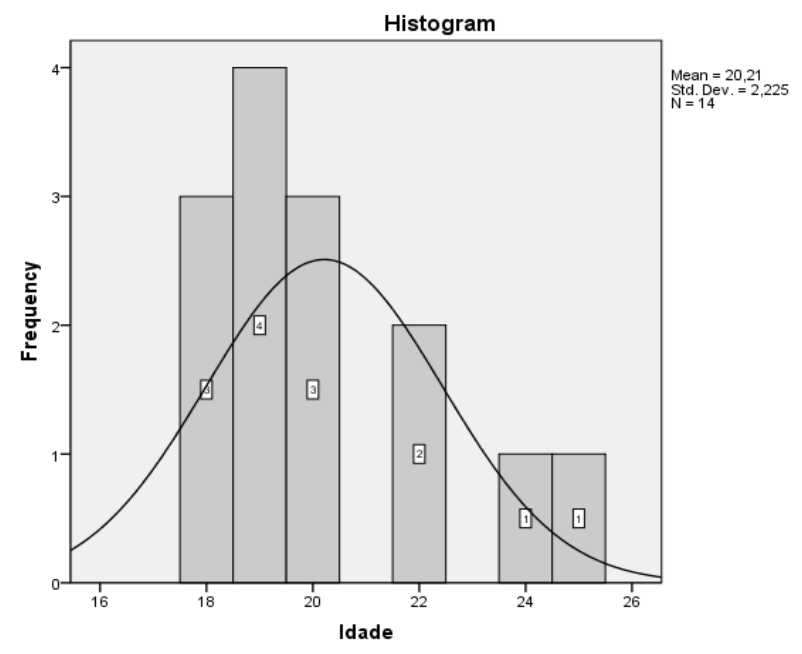

Os informantes selecionados assinaram um termo de consentimento livre e esclarecido e responderam a um questionário que serviu para uma triagem secundária. Além disso, antes da coleta de dados de percepção, cada informante gravou, via software 
PRAAT (BOERSMA; WEENINK, 2013), a leitura de um parágrafo que continha oito itens com contexto para palatalização, isto é, /t/ precedendo [i]. Todos os informantes palatalizaram a plosiva coronal em todos os oito contextos. Desta forma, os dados do questionário e a gravação nos revelam que: (i) todos os informantes nasceram em Chapecó (SC) ou entrono (Xaxim, Xanxerê, Águas de Chapecó, Pinhalzinho e Guatambú); (ii) todos têm o Português Brasileiro como língua materna; (iii) todos palatalizam as plosivas coronais antes da vogal alta [i]; (iv) o inglês é a única língua estrangeira do grupo; (v) nenhum dos informantes morou em regiões não palatalizadoras do Brasil; e (vi) nenhum do grupo morou em um país falante de língua inglesa.

\section{Sobre as palavras-alvo}

Os instrumentos de percepção contaram com palavras monossilábicas do inglês, iniciadas pela consoante plosiva coronal surda ou pela africada surda, seguidas pelas vogais [I, i:, u:, ॰, U, 3, æ]. Para esse estudo, os estímulos utilizados nos testes de percepção são produções das palavras-alvo, gravadas por um adulto falante nativo de língua inglesa, com 26 anos de idade, nascido nos Estados Unidos da América e residente no Brasil a menos de um ano. A gravação foi feita em estúdio profissional na cidade de Porto Alegre (RS), conferindo uma alta qualidade sonora aos itens utilizados na confecção dos instrumentos de coleta, mantendo-se, assim, um rigor metodológico adequado com a nossa proposta para esse trabalho.

\section{Teste de identificação}

Nas últimas décadas, a pesquisa que se debruça sobre a percepção dos sons tem utilizado um elenco de testes de percepção. No presente trabalho, contudo, adotaremos um dos modelos de testagem mais comuns na literatura da área, o teste de identificação. Grosso modo, em um teste de identificação, um único estímulo (palavra-alvo) é apresentado, e o informante deve classificá-lo como sendo uma das opções apresentadas pelo instrumento. Nesta trabalho, o teste foi elaborado com o software TP (RAUBER; RATO; KLUGE; SANTOS, 2013), antigo TP-S. Optamos pelo TP, pois fora desenvolvido para ser mais facilmente manuseado pelos profissionais que não dominam a linguagem de programação que softwares como o PRAAT (BOERSMA; WEENINK, 
2013) demandam. O software que adotamos permite, além da elaboração de testes de identificação e percepção, uma visualização das respostas para cada estímulo ou de forma cumulativa e os tempos de reação de cada participante. Outra vantagem do TP é que ele randomiza automaticamente a ordem de apresentação dos estímulos a cada início da testagem, reduzindo, assim, qualquer efeito de viés oriundo de um ordenamento estático dos estímulos, bem como efeitos de borda de início e de término de testagem.

O objetivo da tarefa de identificação, neste trabalho, era analisar se os informantes eram capazes de identificar a plosiva coronal [t] aspirada e a africada surda [t $f$ ]. Os estímulos consistiam na pronúncia de uma única palavra como, por exemplo, tick ou chick, apresentada isoladamente. Do arquivo original de áudio, foram recortadas, via software PRAAT (BOERSMA; WEENINK, 2013), 42 palavras, 21 iniciadas por [ $\mathrm{t}^{\mathrm{h}}$ ] e 21 iniciadas por $\left[\mathrm{t} \int\right]$ precedendo os contextos vocálicos mencionados, que foram apresentadas aos informantes. Os 14 estudantes participaram do teste, que foi realizado em sala silenciosa e com headsets com retorno de até $20.000 \mathrm{~Hz}$, resultando na identificação de 588 tokens (294 para [ $\mathrm{t}^{\mathrm{h}}$ ] e 294 para [t $\left.\left.\int\right]\right), 42$ repetições únicas de cada item multiplicadas pelos 14 informantes.

\section{DISCUSSÃO E ANÁLISE DE DADOS}

Como já mencionado, o teste de identificação resultou em 588 tokens. Na Tabela 1, encontramos os dados sobre a média de acertos na tarefa em função dos dois níveis de proficiência considerados. Em linhas gerais, o Teste de correlação Spearman revela uma associação significativa entre os níveis de proficiência e as percentagens de acerto no teste de identificação $\left(\mathrm{r}_{\mathrm{sp}}=0,53, \mathrm{p}=0,04\right)$.

TABELA 1 - Média de acertos na tarefa de identificação

\begin{tabular}{cccc} 
Proficiência & Média & N & DP \\
\hline Básico & $84,00 \%$ & 6 & 10,60 \\
\hline Avançado & $94,00 \%$ & 8 & 3,85 \\
\hline Total & $89,92 \%$ & 14 & 8,90
\end{tabular}

Resgatando os dados acima, a média de acertos total para a tarefa de identificação foi de $89,92 \%$ ( $\mathrm{DP}=8,90)$. Para os acertos em palavras iniciadas em [ $\left.\mathrm{t}^{\mathrm{h}}\right]$, obtivemos uma 
média de 91,79\% (DP = 11,20). Já para palavras iniciadas pela africada [ts], a média foi de 87,86\% (DP = 10,13). Sendo a distribuição normal dos dados $(\mathrm{p}>0,05)$, nossa análise via Teste T Pareado aponta não haver diferença significativa entre as médias de acertos para os dois segmentos aqui analisados $(\mathrm{t}(13)=1,364, \mathrm{p}=0,20)$. Assim, de forma geral, estatisticamente, os informantes obtiveram altos índices de acerto na testagem tanto na identificação das africadas quanto das plosivas aspiradas do inglês. Ainda nesta perspectiva, selecionando-se apenas os informantes classificados como básicos em língua inglesa, o teste de diferença não paramétrico Wilcoxon aponta não haver uma diferença significativa $(Z=-137, p=0,90)$ entre as médias de acerto para as palavras iniciadas pela plosiva (média de $83,33 \%$, DP $=12,30$ ) e para as palavras iniciadas pela africada (média de $85 \%$, DP 13,42). O grupo de falantes avançados, via Wilcoxon, também não sinalizou diferença significativa entre os índices de acerto para os dois segmentos $(Z=-1,715, p=$ 0,87), com médias 91,25\% ( $\mathrm{DP}=5,82)$ e 96,88\% ( $\mathrm{DP}=5,94)$ para os acertos da africada e da plosiva aspirada, respectivamente.

Debruçando-se sobre o número de acertos no teste de identificação, a média de erro dos informantes básicos foi de 10,50 erros $(\mathrm{DP}=5,56$ erros $)$ e a dos falantes avançados, 4,63 erros ( $\mathrm{DP}=3,70$ erros). Sendo a distribuição dos dados normal ( $\mathrm{p}>$ $0,05)$, nossa testagem via Teste $\mathrm{T}$ Independente aponta uma diferença significativa $(\mathrm{t}(12)$ $=2,136, \mathrm{p}=0,05)$ entre o desempenho na testagem em relação aos níveis aqui analisados, sendo que os falantes avançados tiveram uma melhor performance nesta testagem perceptual, como seria, hipoteticamente, de se esperar. Ainda neste sentido, o teste de correlação de Spearman revela a existência de uma associação significativa entre nível de proficiência e desempenho na testagem.

Contudo, analisando-se a diferença entre os níveis de proficiência e os índices de erros nos contextos vocálicos mencionados, nossa testagem, via teste não paramétrico Mann-Whitney, revela haver diferença significativa na média de erros em função do nível de proficiência apenas quando os segmentos a serem identificados estavam no contexto que antecede a vogal alta [i:] do inglês $(U=-2.189, p=0,03)$. Para os demais contextos, não foram reveladas diferenças significativas $(\mathrm{p}>0,05)$.

À luz desta discussão, primeiramente, nossos dados corroboram os primados de Best e Tyler (2007) e de Flege (1995) de que a maior experiência em uma L2 levaria a uma maior habilidade perceptual nesta língua, já que atestamos que falantes avançados obtiveram as maiores médias de acerto em comparação aos falantes básicos.

Complementando nossa análise, Yavas e Wildermuth (2006) apontam que a 
aspiração aumentaria de acordo com a altura da vogal. Ainda neste sentido, França (2011) relata, também, que vogais altas podem apresentar uma aspiração mais fácil de ser notada. Todavia, nossos dados traduzem índices mais altos de erros para a vogal [i:] em relação às demais vogais. Neste prisma, acreditamos que a origem desses índices seria o fato de todos os informantes apresentarem, como variável controlada, em L1, a produção da africada palatal surda antes da vogal alta não arredondada. Nesta perspectiva, podemos pensar que os informantes básicos, dado o grau de similaridade, estimam os segmentos testados como tokens de um mesmo som nativo, isto é, categorizariam africada e plosiva aspirada antes de vogal alta não arredondada em uma mesma categoria, conforme previsto para uma discriminação pobre por Best e Tyler (2007). Destarte, confirmamos a nossa hipótese de que falantes nativos palatalizantes do PB, acostumados com o chiamento antes da vogal alta não arredondada, apresentariam índices distintos na identificação da aspiração do inglês para contextos que seguem vogal alta e para os demais contextos vocálicos.

\section{CONSIDERAÇÕES FINAIS}

Este trabalho analisou a percepção de [ $\left.\mathrm{t}^{\mathrm{h}}\right]$ e [t $\left.\int\right]$ do inglês-L2 por falantes palatalizantes do Oeste de Santa Catarina. Embora com rigores metodológicos limitados quanto à natureza dos estímulos para a elaboração das tarefas e quanto ao número de informantes, o presente estudo sugere algumas considerações, mesmo que preliminares. Não vemos apenas diferenças entre desempenho em função dos níveis de proficiência, mas também levando-se em consideração o contexto vocálico que segue o item a ser identificado. Nossa hipótese de que as vogais altas não arredondadas do inglês apresentariam índices distintos de percepção em relação às demais vogais foi confirmada.

Os próximos passos de pesquisa incluem a reformulação dos testes com um maior número de locutores bom como computar os dados de testes de discriminação já realizados. Além disso, há a necessidade de se reaplicar os testes em um maior número de sujeitos $(\mathrm{N}>30)$. Em adição, pretendemos aplicar os testes em falantes não palatalizadores, para que nossos resultados possam ser comparados. 


\section{REFERÊNCIAS}

ALBANO, E. C. O Gesto e suas Bordas: esboço de Fonolgia Acustico-Articulatória do Português Brasileiro. Campinas: Mercado de Letras/ São Paulo: FAPESP, 2001. ALLAN, D. Oxford Placement Test 1. Oxford: Oxford University Press, 2004. ALVES, U.; SCHWARTZHAUPT, B.; BARATZ, A.; A percepção dos padrões de VOT longo do inglês por aprendizes brasileiros. In: III SAF - Seminário de Aquisição Fonológica, 2011, Pelotas-RS. III Seminário de Aquisição Fonológica - Programação e Resumos. Pelotas-RS : Editora da UFPel, 2011. p. 88-89.

BATTISTI, E.; GUZZO, N.B. Palatalização das oclusivas alveolares: O caso de Chapecó. In: BISOL, L.; COLLISCHONN, G. (Orgs.) Português no sul do Brasil: Variação fonológica. Porto Alegre: EDIPUCRS, 2009.

BEST, C. T. A direct realist view of cross-language speech perception. In: STRANGE, W. (Ed.). Speech perception and linguistic experience: issues in cross-language research. Timonium, MD: York Press, 1995, p. 171-204.

BEST, C.; TYLER, M. Nonnative and second- language speech perception: commonalities and complemen- tarities. In: BOHN, O.; MUNRO, M.. Language Experience in Second Language Speech Learning: In honor of James Emil Flege. Amsterdam: John Benjamins, 2007. p. 13-34.

BOERSMA, P.; WEENINK, D. PRAAT: doing phonetics by computer. Versão 5.3.01 (programa computacional). Disponível em: http://www.praat.org.

BROWMAN, C.; GOLDSTEIN, L. Gestural structures: Distinctiveness, phonological processes and historical change. In: MATTINGLY, I.; STUDDERT-KENNEDY, M. (Eds), Modularity and the motor theory of speech perception: Proceedings of a conference to honor Alvin M. Liberman (pp. 313-338). Hillsdale, NJ: Lawrence Erlbaum Associates, 1990.

Articulatory Phonology: an overview In: Phonetica, 1992, n. 49, p. 155-180.

CHO T.; LADEFOGED, P. Variations and universals in VOT. Fieldwork studies of targeted languages V: UCLA Working Papers in Phonetics, 95, 1999.

CRISTÓFARO-SILVA, T. Fonética e fonologia do português: roteiro de estudos e guia de exercícios. 4. ed. São Paulo: Contexto, 2001. 261 p.

FLEGE, J. Second language speech learning: Theory, findings and problems. In: STRANGE, W. (Ed.). Speech perception and linguistic experience: Theoretical and methodological issues. Timonium, MD: York Press, 1995, p. 233-277.

GUIMAR ÃES, D. Sequencias de (sibilante + africada alveopalatal) no português falado em Belo Horizonte. Belo Horizonte: UFMG. Dissertação (Mestrado em Letras), 2004.

JOHNSON, K. Acoustic and Auditory Phonetics. 2.ed. Oxford: Blackwell, 2003.MACHADO, 2003.

KUPSKE, F. Opacidade Fonológica em português: uma resposta via teoria da otimidade conexionista para uma questão cartesiana. Santa Maria: UFSM. Dissertação. Mestrado em Letras, 2011.

LADEFOGED, P.; MADDIESON, I. The Sound of World's Languages. Oxford: Blackwell, 1996.

LISKER, L; ABRAMSON, A. S. A cross-language study of voicing in initial stops: acoustical measurements. Word, v. 20, p. 384-422, 1964.

MACWHINNEY, B. Language Emergence. In: BURMEISTER, P.; PISKE T.; RHODE, A. (Ed.) An integrated view of language development. Papers in honor of Henning Wode. Trier: Wissenchaftliche Verlag, 2002. p.17-42. 
MACWHINNEY, B. Emergentism - Use Often and With Care. Applied Linguistics, n. 27, p. 729-740, 2006.

RAUBER, A; RATO, A; KLUGE, D; SANTOS, G; FIGUEIREDO, M. TP - Teste de percepção, 2013. Programa computacional. Disponível em www.worken. com.br/tp_regfree.php

REIS, M S; NOBRE-OLIVEIRA, D; RAUBER, A.S. Effects of perceptual training on the identification and production of the English voiceless plosives by Brazilian EFL learners. In: Proceedings of the New Sounds Fifth International Symposium on the Acquisition of a Second Language Speech. Florianópolis, nov. 2007. p. 25-28.

REIS, M. S, NOBRE-OLIVEIRA, D. Effects of perceptual training on the identification and production of the English voiceless plosives aspiration by Brazilian EFL learners. New Sounds 2007. Proceedings of the Fifth International Symposium on the Acquisition of Second Language Speech, 398-407, 2007.

SEIDENBERG, M.; ZEVIN, J. Connectionist Models in Developmental Cognitive Neuroscience: critical periods and the paradox of success. In: MUNAKATA, Y; SILVA, A. Pela incorporação de informação fonética aos modelos fonlógicos. Revista Letras, v. 60, p. 319 - 333. Curitiba: Editora UFPR, 2003.

YAVAS, M.; WILDERMUTH, R. The effects of place of articulation and vowel height in the acquisition of English aspirated stops by Spanish speakers. IRAL, v. 44, p. 251-263, 2006.

ZIMMER, M.C.; SILVEIRA, R.; ALVES, U.K. Pronunciation instruction for Brazilians: bringing theory and practice together. Cambridge: Cambridge Scholars Publishing, 2009.

ZIMMER, M. Cognição e aprendizagem de L2: uma abordagem conexionista. In: MACEDO, A. C.; FELTES, H.; FARIAS, E.M. (Orgs.). Cognição e Linguística: Territórios, Mapeamentos e Percursos. Porto Alegre/Caxias do Sul: EDIPUCRS/EDUCS, 2008. p. 229-248.

ZIMMER, M.; ALVES, U.; SILVEIRA, R. A aprendizagem de L2 como processo cognitivo: a interação entre conhecimento explícito e implícito. Nonada (Porto Alegre), v. 9, p. 157-174, 2006.

ZIMMER, M. C. A transferência do conhecimento fonético-fonológico do português brasileiro (L1) para o inglês (L2) na recodificação leitora: uma abordagem conexionista. 2004. 187 folhas. Tese - Doutorado em Letras. PUCRS, Porto Alegre, 2004. 